\title{
Microwave Ablation of Benign Thyroid Nodules: Effects on Systemic Inflammatory Response
}

\author{
Mehmet Sercan Erturk' ${ }^{1}$, Bulent Cekic ${ }^{2}$ and Mehmet Celik ${ }^{3}$ \\ ${ }^{1}$ Department of Endocrinology and Metabolism, University of Health Sciences, Antalya Training and Research Hospital, Antalya, Turkey \\ ${ }^{2}$ Department of Radiology, University of Health Sciences, Antalya Training and Research Hospital, Turkey \\ ${ }^{3}$ Department of Endocrinology and Metabolism, Trakya University Medical Faculty, Edirne, Turkey
}

\begin{abstract}
Objective: To explore the alterations of neutrophil-to-lymphocyte ratio (NLR) as a systemic inflammatory marker after microwave ablation for benign thyroid nodules.

Study Design: A descriptive study.

Place and Duration of Study: Department of Radiology, Antalya Training and Research Hospital, from December 2018 to June 2019.

Methodology: Demographic data of the patients, ultrasonographic features of the nodules, thyroid function tests of the patients, leukocyte, neutrophil, lymphocyte values, and thyroid nodule volumes of the patients before and after the procedure were recorded. NLR and volume reduction ratio (VRR \%) of 35 patients with thyroid nodules were compared before and after microwave ablation therapy of the thyroid nodules.

Results: The nodule volume decreased from $23.89 \pm 15.44$ cc to $11.57 \pm 8.65$ cc at two months and to $7.79 \pm 5.74$ cc at six months. The VRR\% increased from $38.65 \pm 16.82$ to $63.16 \pm 14.19$ at three months and to $68.29 \pm 11.80$ at six months. The mean value of NLR decreased from $2.28 \pm 0.86$ to $1.78 \pm 0.54$. ROC curve analysis suggested that the optimum pre-NLR cut-off point for $50 \%$ VRR success was 2.50 with the specifity and sensitivity of 0.67 and 0.50 .

Conclusion: After ultrasound-guided microwave ablation of benign thyroid module, VRR percentage increased significantly, while the inflammatory marker NLR value decreased.
\end{abstract}

Key Words: Biomarker, Inflammation, Microwaves, Neutrophil-to-lymphocyte ratio, Thyroid nodule.

How to cite this article: Erturk MS, Cekic B, Celik M. Microwave Ablation of Benign Thyroid Nodules: Effects on Systemic Inflammatory Response. J Coll Physicians Surg Pak 2020; 30(07):694-700.

\section{INTRODUCTION}

Thyroid nodules constitute the most common disease group of the thyroid gland. The prevalence of thyroid nodules increases progressively in women and men with aging. In particular, after the age of 50 years, it increases to $18.0 \%$ for women and $14.5 \%$ for men ${ }^{1,2}$ In the last 20 years, noninvasive or minimally invasive thermal ablation methods have been developed for the treatment of benign nodular thyroid diseases. These methods are percutaneous ethanol injection (PEE), percutaneous thermal laser ablation (PLA), radiofrequency ablation (RFA), microwave ablation (MWA) and high intensity focused ultrasound (HIFU). ${ }^{3}$

Correspondence to: Mehmet Sercan Erturk, Department of Endocrinology and Metabolism, University of Health Sciences, Antalya Training and Research Hospital, Antalya, Turkey

E-mail: msercanerturk@gmail.com

Received: April 04, 2020; Revised: May 29, 2020;

Accepted: July 02, 2020

DOI: https://doi.org/10.29271/jcpsp.2020.07.694
Systemic inflammatory response syndrome (SIRS) is defined as inflammation in the body against non-infectious causes such as infection or autoimmune diseases, pancreatitis, vasculitis, thromboembolism, burns, surgeries. SIRS diagnostic criteria are formed with the use of parameters, including body temperature, heart rate, respiration rate and leukocyte counts. ${ }^{4}$ Each criterion in this syndrome emerges in response to the inflammatory response. Recently, the number of studies examining neutrophil-to-lymphocyte ratio (NLR) as an indicator of inflammatory response has increased as an easy and cost-effective method for this purpose. ${ }^{5,6}$ It has been suggested in literature that NLR can be used in patients who have undergone RFA in the follow-up of systemic inflammatory response, but there are no studies examining NRL change or correlation after MWA. ${ }^{6}$ The aim of this study was to explore alterations of systemic inflammatory marker, such as neutrophil-to-lymphocyte ratio (NLR), after microwave ablation in patients with benign thyroid nodules.

\section{METHODOLOGY}

Informed consents were obtained from all patients who underwent microwave ablation for benign thyroid nodules. This descriptive study protocol was approved by the University of 
Health Sciences, Antalya Training and Research Hospital Ethics Committee (Ethics Committee approval number and date: 27/9 \& 26/12/2019). Thirty-five patients between December 2018 and June 2019 eligible for the study were included. Inclusion criteria of this study were symptomatic (globus sensation, swallowing complaints, cosmotic concerns) euthyroid solid (solid component $>80 \%$ ) nodules; ineligibility or refusal to undergo surgery; and histopathological benign status (Bethesda category 2 nodules). The exclusion criteria were pregnancy under age 18 years, bleeding disorders; malignant thyroid nodule cytology; and nodules with high risk sonographic patterns such as taller-than-wide shape, irregular border, and microcalcification.

Demographic data of the patients, ultrasonographic features of the nodules, thyroid function tests of the patients, leukocyte, neutrophil, lymphocyte values, and thyroid nodule volumes of the patients before and after the procedure were recorded. Serum thyroid-stimulating hormone (TSH), Free T3 (FT3) and Free T4 (FT4) was measured by Unicel TM DxI 800 Access Immunoassay System, Beckman Coulter Inc., Brea, CA, USA. The normal ranges were as follows: free T4, 0.61-1.1 ng/dL; free T3, 2.5-3.9 pg/mL; TSH, 0.34-5.86 $\mu \mathrm{lU} / \mathrm{mL}$. White blood cell (WBC), neutrophil and lymphocyte counts were measured using an automated whole blood analyzer (Abbott Cell Dyn, IL, USA). The NLR was calculated by dividing the absolute neutrophil count by the absolute lymphocyte count. The difference in the NLR ( $\triangle N L R$ ) was computed by subtracting preoperative NLR (Pre-NLR) from the NLR value after microwave ablation treatment (Post-NLR). The values smaller than the standard deviation of the $\triangle N L R$ was defined as decreased NLR while greater or equal ones donated as increased NLR. The rest of the patients was handled as stable NLR. ${ }^{7}$

The ultrasonography examination, 5-14 MHz linear probe with a real time ultrasound system (Aplio 500,Toshiba Medical Systems, Tokyo, Japan) was used to evaluate volume, diameters, vascularity, echogenicity, and composition of the nodules before procedure and 1, 3, 6 months after procedure. Three orthogonal diameter (the largest and two other perpendicular diameters) of nodules were measured before ablation. The volume of nodules was calculated with the following equations: $\mathrm{V}=\pi \mathrm{abc} / 6$ (V: volume, $\pi ; 3.14159$ a: the largest diameter; $b$ and $\mathrm{c}$ : the other two perpendicular diameters). The volume reduction ratio (VRR \%) was calculated based on the equation: [Baseline volume-volume at visit]/[Baseline volume] 100.

Microwave ablation system (ECO-100AI3), consisting of a microwave generator producing $30-40 \mathrm{~W}$ of power at $2450 \mathrm{MHz}$ either continuously or in a pulse; a flexible cable, an internally-cooled antenna with a $3.5 \mathrm{~mm}$ active tip and $10 \mathrm{~cm}$ shaft length, was used. Ablation was performed as an outpatient treatment under local anesthesia without sedation. After determination of appropriate puncture side, local anesthesia, with 50/50 mixture of $2 \%$ lidocaine and saline, was performed along the puncture pathway from the skin to the thyroid capsule. The hydrodissection technique proposed by Yue et al. was used, ${ }^{8}$ and a mixture of $0.9 \%$ lidocaine and physiological saline was infused into the surrounding thyroid capsule to protect vital structures adjacent to the thyroid nodule. A small incision about $2 \mathrm{~mm}$ in length was made and internally cooled microwave ablation antenna was positioned under ultrasound guidance and moving shot technique was used. ${ }^{6,8}$ Treatment was stopped when all of the nodule was covered with hyperechoic echo.

Power analysis was performed using $\mathrm{G} *$ Power (v3.1.9) programme to determine the sample size. ${ }^{9} \mathrm{Wu}$ et al. reported the mean nodule volume before treatment was $6.61 \pm 4.65 \mathrm{ml}$, while the nodule volume after microwave ablation was $4.03 \pm$ $3.30 \mathrm{ml}^{10}$ The effect size (df) of that study was determined as 0.62 . Supposing a true difference in the mean response of that study subjects was 3.78, 35 cases, were inducted to be able to reject the null hypothesis that the response difference was zero with probability (power) 0.97. Typelerrorprobability associated with this test of this null hypothesis was 0.05 . All the statistical tests were carried out by SPSS ${ }^{\circledR} 20.0$ (Statistical Packages for Social Sciences; SPSS Inc, Chicago, Illinois, USA). The Shapiro-Wilk test was used to evaluate the normality of the data. Qualitative variables were expressed as frequencies and percentages and quantitative as mean \pm S.D and Median (IQR). The comparisons between the dependent groups were performed by Wilcoxon signed-rank test where the normalities of the differences are not met. In other words, paired t-test was used. A one-way repeated measures ANOVA was conducted to determine whether there were statistically significant differences in the nodule volumes in six months. Continuous variables between dependent groups were compared using the Mann-Whitney U-test. Univariate and multivariate logistic regression were used to assess factors leading to differentiation on the NLR differences. To get a better understanding the predictive role of pre-NRL on volume reduction of the nodules, a receiver operating characteristic curve (ROC) was facilitated to determine the sensitivity and specify of pre-NLR on the volume reduction rate percent (VRR\%) of nodules after six months of the treatment with microwave ablation by referencing the $50 \%$ VRR as limiting success.

\section{RESULTS}

The mean age of the patients included in the study was 50.59 \pm 14.35 years. The total number of patients was 35 . Nineteen (54.3\%) of the patients were females and $16(45.7 \%)$ were males. The microwave ablation elicited statistically significant changes in nodule volume (cc) over time $(p<0.001)$, with nodule volume decreasing from $23.89 \pm 15.44 \mathrm{cc}$ to 11.57 \pm 8.65 at two months and to $7.79 \pm 5.74$ ccat six months.

Post-hoc analysis with Bonferroni adjustment revealed that after microwave ablation, decrease in the nodule volumes for 1, 2, 3 and 6 months were $8.43 \mathrm{cc}(95 \% \mathrm{Cl}, 6.60$ to 10.27 , $\mathrm{p}<0.001), 12.33 \mathrm{cc}(95 \% \mathrm{Cl}, 9.49$ to $15.16, \mathrm{p}<0.001), 14.74 \mathrm{cc}$ $(95 \% \mathrm{Cl}, 11.40$ to $18.08, \mathrm{p}<0.001)$, and $16.11 \mathrm{cc}(95 \% \mathrm{Cl}, 12.42$ to $19.80, p<0.001$ ), respectively. It is noted that there was a significant decrease in volume over time. The result revealed 
that there were statistically significant changes between mean VRR percentage over time after microwave ablation performed $(p<0.001)$. The VRR percentage increased from $38.65 \pm 16.82 \%$ to $63.16 \pm 14.19 \%$ at three months and to $68.29 \pm 11.80 \%$ at six months. Post-hoc analysis with a Bonferroni adjustment demonstrated that VRR percentage was increased significantly from the prior of the microwave ablation to 1 month $38.65(95 \% \mathrm{Cl}, 30.12$ to $47.19, \mathrm{p}<0.001), 2$ months $53.61(95 \% \mathrm{Cl}, 45.75$ to $61.46, \mathrm{p}<0.001)$, 3 months $63.16(95 \% \mathrm{Cl}, 55.96$ to $70.36, p<0.001)$, and 6 months 68.29 $(95 \% \mathrm{Cl}, 62.30$ to $74.28, p<0.001)$. There was a significant increase in the VRR percentage over time. The change in nodule volume and VRR percentage as a function of time (months) werealso shown in Figure la and Figure 1b.
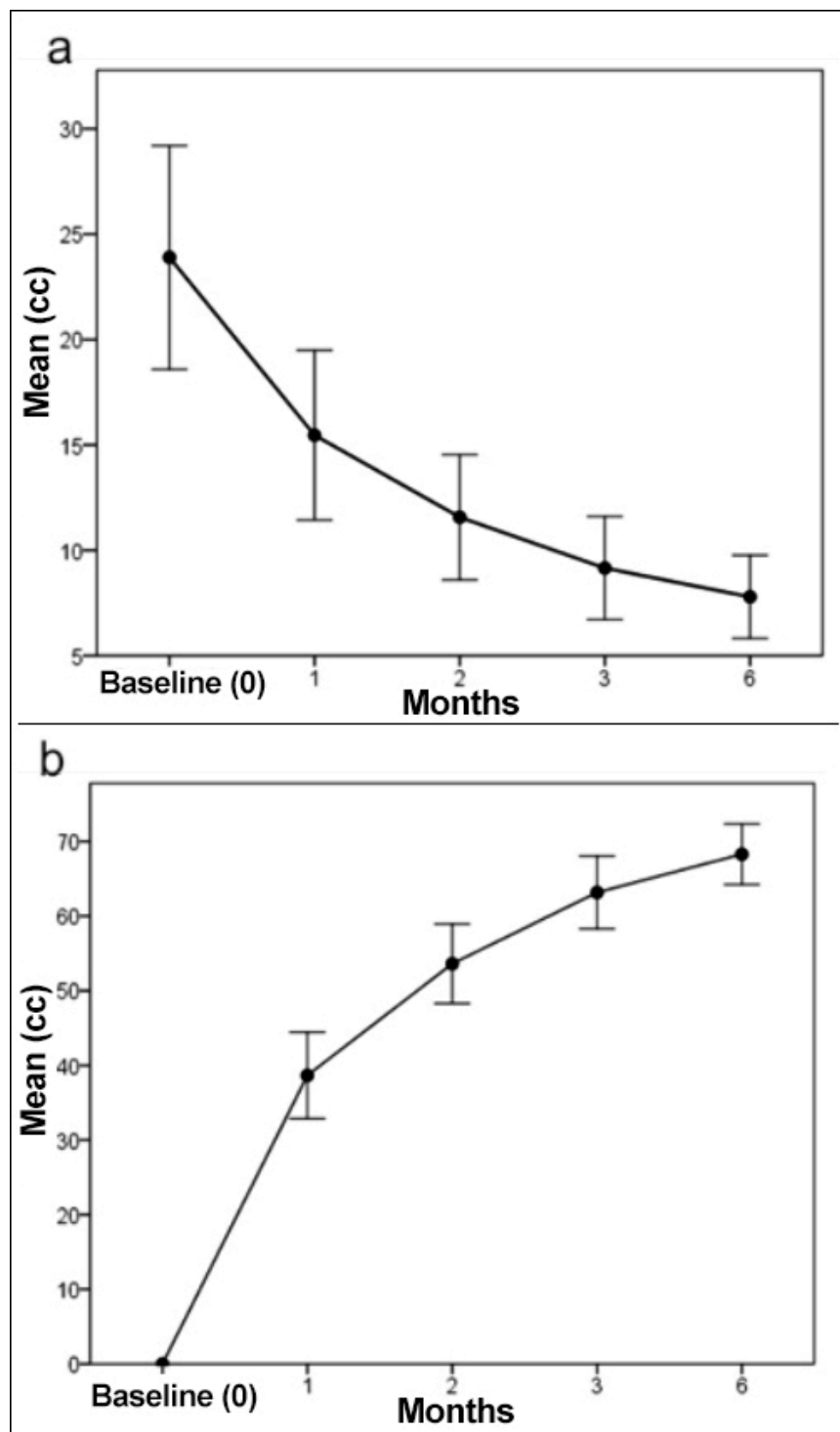

Figure 1: (a) Continuous decrease in the nodule volume after microwave ablation. Comparison of the volumes of nodules at baseline (month: 0 time of microwave ablation) and at follow-up after treatment. The nodule volume at pre, 1-, 2-, 3-, 6-months was $23.89 \pm 15.44,15.46 \pm 11.73,11.57$ $\pm 8.65,9.15 \pm 7.13,7.79 \pm 5.74 \mathrm{cc}$, respectively; (b) Increase in the VRR percentage at baseline and at follow-up after treatment. The VRR percentage at 1-, 2-, 3-, 6-months was $38.65 \pm 16.82,53.60 \pm 15.49,63.16$ $\pm 14.19,68.29 \pm 11.80 \%$, respectively.
Table I: Baseline and changes of the values after MW ablation at 6 months follow-up.

\begin{tabular}{|c|c|c|c|}
\hline & $\begin{array}{l}\text { Pre-ablation } \\
\text { (n 35) }\end{array}$ & $\begin{array}{l}\text { After-ablation } \\
\text { (n 35) }\end{array}$ & p-value \\
\hline Volume (cc) & & & $<0.001 *$ \\
\hline Mean \pm SD & $23.89 \pm 15.44$ & $7.79 \pm 5.74$ & \\
\hline WBC $\left(10^{3} / \mu l\right)$ & & & $0.009 *$ \\
\hline Median (IQR) & $7.60(7.00-8.70)$ & $7.00(6.50-8.20)$ & \\
\hline Neutrophil $\left(10^{3} / \mu \mathrm{l}\right)$ & & & $<0.001^{*}$ \\
\hline Mean \pm SD & $4.75 \pm 1.28$ & $4.25 \pm 1.18$ & \\
\hline Lymphocyte $\left(10^{3} / \mu \mathrm{l}\right)$ & & & 0.003* \\
\hline Median (IQR) & $2.10(1.70-2.70)$ & $2.50(2.00-2.80)$ & \\
\hline NLR & & & $<0.001 *$ \\
\hline Mean \pm SD & $2.28 \pm 0.86$ & $1.78 \pm 0.54$ & \\
\hline FT3 $(2.5-3.9 \mathrm{pg} / \mathrm{mL})$ & & & 0.147 \\
\hline Median (IQR) & $3.48(3.09-3.99)$ & $3.48(3.39-3.96)$ & \\
\hline FT4 $(0.61-1.1 \mathrm{ng} / \mathrm{dL})$ & & & $0.001^{*}$ \\
\hline Median (IQR) & $0.82(0.71-0.88)$ & $0.87(0.81-0.93)$ & \\
\hline TSH $(0.34-5.86 \mu \mathrm{IU} / \mathrm{mL})$ & & & 0.195 \\
\hline Median (IQR) & $0.96(0.25-1.69)$ & $0.60(0.37-1.34)$ & \\
\hline \multicolumn{4}{|c|}{ 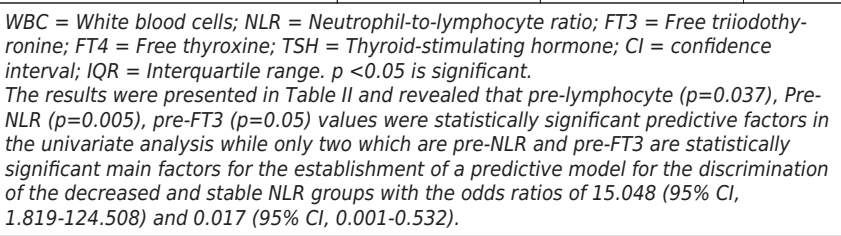 } \\
\hline
\end{tabular}

Table I displays the change in the monitored parameters after microwave ablation treatment to the patients with benign thyroid nodules. After microwave treatments, the mean volume of the nodules decreased from $23.89 \pm 15.44$ before ablation to $7.79 \pm 5.74(p<0.001)$ in six months follow-up. Likewise, a highly statistically significant decrease in the mean values of neutrophil and NLR from $4.75 \pm 1.28$ and $2.28 \pm 0.86$ to 4.25 \pm 1.18 and $1.78 \pm 0.54$ was observed, respectively $(p<0.001)$. Before the ablation, the median Lymphocyte and FT4 were 2.10 (IQR, 1.70-2.70) and 0.82 (IQR, 0.71-0.88). Their median values significantly increased to 2.50 (IQR, 2.00-2.80) and 0.87 (IQR, $0.81-0.93$ ), respectively, while the median WBC 7.60 (IQR, $7.00-8.70)$ decreased to 7.00 (IQR, 6.50-8.20) $(p=0.009)$. On the other hand, there was no significant difference in the FT3 and TSH values before and after microwave ablation.

Multivariate logistic test results have revealed the predictive role of pre-NRL together with the pre-FT3. To geta better understanding the predictive role of pre-NRL on volume reduction of the nodules, a receiver operating characteristic curve (ROC) was facilitated to determine the sensitivity and specify of preNLR on the volume reduction rate percent (VRR\%) of nodules after six months of the treatment with microwave ablation by referencing the $50 \%$ VRR as limiting success. The ROC curve analysis results suggested the optimum pre-NLR cut-off point as 2.50 with the specifity and sensitivity of 0.67 and 0.50 . The AUC value for NLR was $0.606(95 \% \mathrm{Cl}, 0.43,0.79)$ (Figure 2). Subsequently, considering the found cut-off NLR value, patients were divided into two groups as NLR $>2.50$ and NLR $<2.50$ (Table III). 
Table II. Univariate and multivariate logistic regression analyses of the variables predicting stable and decreased NLR.

\begin{tabular}{|c|c|c|c|c|c|c|}
\hline & $\begin{array}{l}\text { Univarite } \\
\text { odds ratio }\end{array}$ & $95 \% \mathrm{Cl}$ & p-value & $\begin{array}{l}\text { Multivare } \\
\text { odds ratio }\end{array}$ & $95 \% \mathrm{Cl}$ & p-value \\
\hline Age & 0.991 & $0.940-1.045$ & 0.747 & & & \\
\hline Gender & 2.000 & $0.410-9.757$ & 0.391 & & & \\
\hline Pre-WBC & 1.191 & $0.703-2.018$ & 0.516 & & & \\
\hline Post-WBC & 1.133 & $0.688-1.864$ & 0.624 & & & \\
\hline Pre-neutrophil & 1.768 & $0.908-3.444$ & 0.094 & & & \\
\hline Post-neutrophil & 0.896 & $0.449-1.789$ & 0.756 & & & \\
\hline Pre-lymphocyte & 0.123 & $0.017-0.884$ & $0.037^{*}$ & 0.439 & $0.020-9.406$ & 0.598 \\
\hline Post-lymphocyte & 0.438 & $0.104-1.842$ & 0.26 & & & \\
\hline Pre-NLR & 7.468 & $1.823-30.583$ & $0.005^{*}$ & 15.048 & $1.819-124.508$ & $0.012 *$ \\
\hline Post-NLR & 1.751 & $0.442-6.942$ & 0.425 & & & \\
\hline Pre-volume & 0.994 & $0.944-1.046$ & 0.812 & & & \\
\hline Pre-FT3 & 0.162 & $0.026-1.000$ & $0.05^{*}$ & 0.017 & $0.001-0.532$ & $0.020 *$ \\
\hline Post-FT3 & 0.728 & $0.182-2.905$ & 0.653 & & & \\
\hline Pre-FT4 & 0.729 & $0.011-46.362$ & 0.881 & & & \\
\hline Post-FT4 & 10.42 & $0.293-370.852$ & 0.198 & & & \\
\hline Pre-TSH & 1.322 & $0.617-2.831$ & 0.473 & & & \\
\hline Post-TSH & 0.717 & $0.246-2.089$ & 0.542 & & & \\
\hline
\end{tabular}

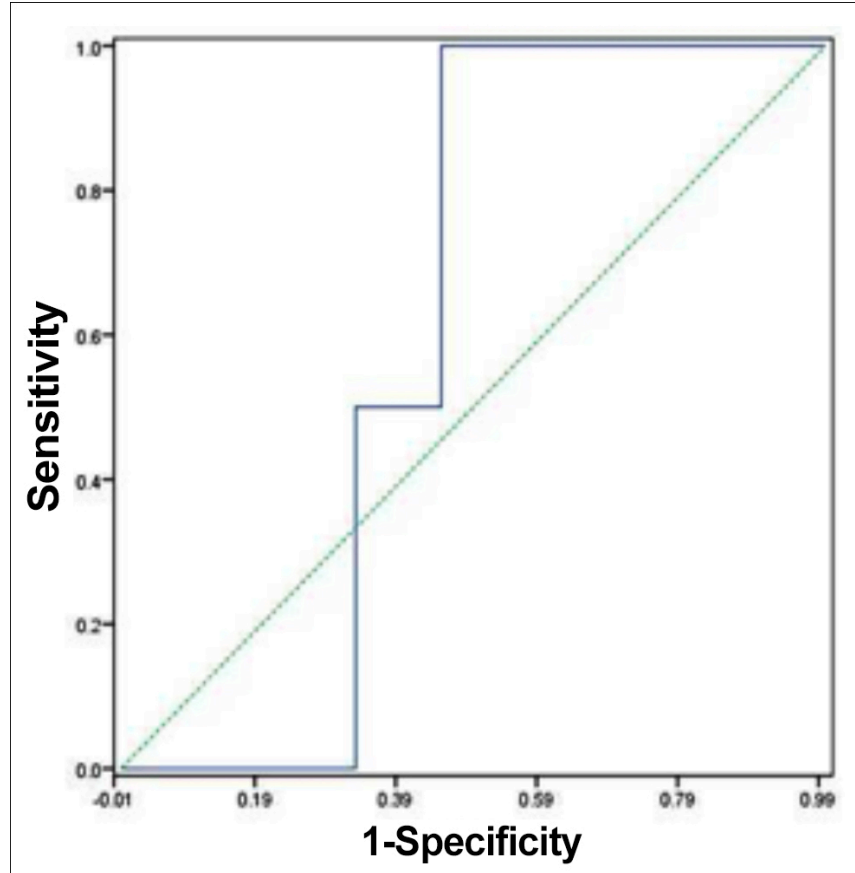

Figure 2: Receiver operating characteristic curves for preoperative NLR value of $\mathbf{2 . 5 0}$ (VRR $\mathbf{5 0 \%}$ ).

There was a statistically significant increase in the median values of the pre-neutrophil $(p=0.008)$, pre-NRL $(p<0.001)$ and post NRL $(p<0.001)$ in NLR $>2.5$ group than NLR $<2.5$ group. On the other hand, a statistically significant decrease was observed for pre-lymphocyte $(p<0.001)$ and post-lymphocyte $(p=0.009)$ in NLR>2.5 group than NLR $<2.5$ group.

\section{DISCUSSION}

Surgical intervention in benign thyroid nodules is often followed by disadvantages and complications such as scar formation, nerve damage, hypoparathyroidism. ${ }^{2}$ Today, minimally invasive ablation methods are used to treat benign thyroid nodules. Among these techniques, MWA is a local thermal ablation method developed as a new treatment modality for benign thyroid nodules. In addition, it is considered to be a good therapeutic minimally invasive method because of its advantages such as fast heating rate, strong coagulation ability, and large ablation area. ${ }^{11}$ The results of our study demonstrated that the MWA significantly decreased the treated nodule volumes after six months of its application. Regarding this result, a former study showed that MWA heats the tissue to a cytotoxic level. ${ }^{12} \mathrm{At}$ this warming level, cell death occurs after coagulation necrosis. Then coagulative necrosis is eliminated with the help of the immune system of the patient with the help of macrophages, lymphocytes and neutrophils. ${ }^{13}$

Neutrophil-to-lymphocyte ratio is a reliable and inexpensive marker for examining the systemic inflammatory response. ${ }^{14}$ 
Table III. Comparison between the parameters of patients with preoperative NLR cut-off value of 2.5 in terms of their $50 \%$ VRR success after six months post microwave ablation.

\begin{tabular}{|c|c|c|c|c|c|}
\hline & \multicolumn{4}{|c|}{ NLR } & \multirow{3}{*}{ p-value } \\
\hline & \multicolumn{2}{|c|}{$<2.5(n=23)$} & \multicolumn{2}{|c|}{$>2.5(n=12)$} & \\
\hline & Median & IQR & Median & IQR & \\
\hline Pre-WBC & 7.50 & $6.67-8.70$ & 7.85 & $7.00-8.95$ & 0.566 \\
\hline Post-WBC & 6.95 & $6.40-8.10$ & 7.45 & $6.90-8.50$ & 0.366 \\
\hline Pre-neutrophil & 4.05 & $3.70-5.40$ & 5.55 & $4.85-6.15$ & $0.008 *$ \\
\hline Post-neutrophil & 4 & $3.40-4.70$ & 4.3 & $3.70-5.05$ & 0.130 \\
\hline Pre-lymphocyte & 2.35 & $2.00-2.70$ & 1.65 & $1.40-1.90$ & $<0.001^{*}$ \\
\hline Post-lymphocyte & 2.6 & $2.30-3.00$ & 1.95 & $1.70-2.50$ & 0.009* \\
\hline Pre-NLR & 1.92 & $1.29-2.14$ & 3.28 & $2.87-3.49$ & $<0.001^{*}$ \\
\hline Post-NLR & 1.56 & $1.33-1.74$ & 2.19 & $1.86-2.53$ & $<0.001^{*}$ \\
\hline Pre-volume & 17.1 & $11.50-32.00$ & 19.7 & $16.00-31.30$ & 0.614 \\
\hline Pre-FT3 & 3.48 & $3.28-3.99$ & 3.39 & $3.07-3.88$ & 0.602 \\
\hline Post-FT3 & 3.48 & $3.39-3.95$ & 3.53 & $3.39-3.96$ & 0.986 \\
\hline Pre-FT4 & 0.81 & $0.77-0.87$ & 0.85 & $0.70-0.88$ & 0.773 \\
\hline Post-FT4 & 0.86 & $0.81-0.91$ & 0.88 & $0.81-0.95$ & 0.876 \\
\hline Pre-TSH & 1.04 & $0.40-1.50$ & 0.68 & $0.05-1.39$ & 0.251 \\
\hline Post-TSH & 0.84 & $0.40-1.44$ & 0.51 & $0.09-1.02$ & 0.073 \\
\hline VRR\% & 69.40 & $63.41-79.90$ & 60.87 & $57.00-70.57$ & 0.089 \\
\hline
\end{tabular}

Indeed, the potential mechanisms underlying the prognostic significance of neutrophils are reactive oxygen species released by neutrophils, nitric oxide and arginase. In other words, the chemicals released from these neutrophils are thought to increase T-cell suppression and increase angiogenesis. ${ }^{15-17}$

Recently, a number of studies examining neutrophil lymphocyte ratio (NLR) as an indicator of inflammatory response has increased as an easy and cost-effective method for this purpose. The results of these studies indicated that there has been a positive relationship between high NLR and recurrence and mortality rates in gastric, colorectal, hepatocellular, gastric and breast cancers. ${ }^{18-20}$ In the present study, the mean NLR value at the $6^{\text {th }}$ month after microwave ablation significantly decreased compared to the pretreatment NLR mean value. Fagnoni et al. evaluated the NLR after radiofrequency ablation in primary and metastatic liver tumors. ${ }^{20}$ In that study, it has been suggested that NLR can be used as a prognostic factor in cellular anti-tumor immune response. ${ }^{21}$ Janikashvili et al. reported a significant decrease in NLR after radiofrequency ablation in patients with colorectal liver cancer. ${ }^{22}$ They suggested that this condition could be used in treatment follow-up. ${ }^{22}$ Ozcelik et al. determined a cut off value of 3.2 in $86.2 \%$ specificity for NLR as an indicator of lymph node metastasis in preoperative thyroid cancers. ${ }^{23}$ In this study, 2.5 cutoff values were taken for pre-NLR value and VRR relationship was evaluated. Although the volume reduction rate (VRR \%) after MWA was more prominent in patients with NLR $<2.5(\mathrm{VRR} \%=69.40 \%)$ before treatment compared to those with pre-treatment NLR $>2.5($ VRR\% $=60.87 \%)$, this difference was found not to be statistically significant $(p>0.05)$.

Feng et al. reported major complications related to MWA including recurrent laryngeal nerve palsy. In the present study, no major complication was occurred. ${ }^{24}$ This can be explained with hydrodissection that protect the major vessel and nerves. Some patients complained that they had mild pain and tingling sensation during the procedure. There were no situations requiring the procedure to be stopped and the use of painkillers.

There were some limitations in this study. Firstly, this study was retrospective single-centre analysis with short follow-up. Secondly, a single parameter was used for systemic inflammatory response evaluation. The researchers did not correlate NRL with other parameters like C-reactive protein (CRP) and procalcitonin.

\section{CONCLUSION}

This study emphasises the importance of immunomodulatory effects in microwave ablation of thyroid nodules 
as the first study evaluated by NLR. However, it is believed that further research is needed to better understand the changes in cytokines and cellular immune response and to assess the effect of the microwave procedure on this system.

\section{ETHICAL APPROVAL:}

The study was approved by the Institutional Ethics Committee of Antalya Training and Research Hospital, and the ethical approvals were obtained prior to initiation of the research work.

\section{PATIENTS' CONSENT:}

The informed consents were obtained from all patients to publish the data concerning this case.

\section{CONFLICT OF INTEREST:}

Authors declared no conflict of interest.

\section{AUTHORS' CONTRIBUTION:}

MSE: Study design, data collection, statistical analysis, literature review, writing and final review and approval of article.

BC, MC: Study design, data collection, literature review, writing and final review and approval of article.

\section{REFERENCES}

1. Reiners $C$, Wegscheider $K$, Schicha $H$, Theissen $P$, Vaupel $R$, Wrbitzky $R$, et al. Prevalence of thyroid disorders in the working population of Germany: Ultrasonography screening in 96,278 unselected employees. Thyroid 2004; 14(11): 926-32.

2. Haugen BR, Alexander EK, Bible KC, Doherty GM, Mandel SJ, Nikiforov YE, et al. 2015 American thyroid association management guidelines for adult patients with thyroid nodules and differentiated thyroid cancer: The American thyroid association guidelines task force on thyroid nodules and differentiated thyroid cancer. Thyroid 2016; 26(1): 1-133.

3. Ahmed M, Solbiati L, Brace CL, Breen DJ, Callstrom MR, Charboneau JW, et al. Image-guided tumor ablation: Standardisation of terminology and reporting criteria - a 10-year update. Radiology 2014; 273(1):241-60.

4. Bone RC, Balk RA, Cerra FB, Dellinger RP, Fein AM, Knaus $W A$, et al. American college of critical care medicine consensus conference: Definition for sepsis and organ failure and guidelines for the use of innovative therapies in sepsis. Crit Care Med 1992; 20(6):864-74.

5. Zeb A, Khurshid S, Bano S, Rasheed U, Zammurrad S, Khan MS, et al. The role of the neutrophil-to-lymphocyte ratio and platelet-to-lymphocyte ratio as markers of disease activity in ankylosing spondylitis. Cureus 2019; 11(10): e6025.

6. Chen TM, Lin CC, Huang PT, Wen CF. Neutrophil-tolymphocyte ratio associated with mortality in early hepatocellular carcinoma patients after radiofrequency ablation. J Gastroenterol Hepatol 2012; 27(3):553-61.

7. Lee F, Yang PS, Chien MN, Lee JJ, Leung CH, Cheng SP. An increased neutrophil-to-lymphocyte ratio predicts incomplete response to therapy in differentiated thyroid cancer. Int J Med Sci 2018; 15(14):1757-63.

8. Yue W, Wang S, Wang B, Xu Q, Yu S, Yonglin Z, et al. Ultrasound guided percutaneous microwave ablation of benign thyroid nodules: Safety and imaging follow-up in 222 patients. Eur J Radiol 2013; 82(1):11-6.

9. Faul F, Erdfelder E, Lang AG, Buchner A. G*Power 3: A flexible statistical power analysis program for the social, behavioral, and biomedical sciences. Behav Res Methods 2007; 39(2):175-91.

10. Wu W, Gong X, Zhou Q, Chen X, Chen X. Ultrasound-guided percutaneous microwave ablation for solid benign thyroid nodules: Comparison of MWA versus control group. Int J Endocrinol 2017; 2017:9724090.

11. Bressem KK, Adams LC, Vahldiek JL, Erxleben C, Poch F, Lehmann KS, et al. Subregion Radiomics Analysis to Display Necrosis After Hepatic Microwave Ablation-A Proof of Concept Study. Invest Radiol 2020; 55(7):422-9.

12. Lubner MG, Brace CL, Hinshaw JL, Lee FT. Microwave tumor ablation: Mechanism of action, clinical results, and devices. J Vasc Interv Radiol 2010; 21(8 Suppl): S192-S203.

13. Facciorusso A, Di Maso M, Muscatiello N. Microwave ablation versus radiofrequency ablation for the treatment of hepatocellular carcinoma: A systematic review and meta-analysis. Int J Hyperthermia 2016; 32(3):339-44.

14. Hwang SY, Shin TG, Jo IJ, Jeon K, Suh GY, Lee TR, et al. Neutrophil-to-lymphocyte ratio as a prognostic marker in critically-ill septic patients. Am J Emerg Med 2017; 35(2): 234-9.

15. Kim C. Understanding the nuances of microwave ablation for more accurate post-treatment assessment. Future Oncol 2018; 14:1755-64.

16. Gonzalez-Aparicio M, Alfaro C. Influence of interleukin-8 and neutrophil extracellular trap (net) formation in the tumor microenvironment: Is there a pathogenic role? J Immunol Res 2019; 2019:6252138.

17. Saeed SA, Waqar MA, Zubairi AJ, Bhurgri H, Khan A, Gowani SAS, et al. Myocardial ischaemia and reperfusion injury: Reactive oxygen species and the role of neutrophil. J Coll of Physicians Surg Pak 2005; 15(8):507-14.

18. Peng J, Li H, Ou Q, Lin J, Wu X, Lu Z, et al. Preoperative lymphocyte-to-monocyte ratio represents a superior predictor compared with neutrophil-to-lymphocyte and platelet-to-lymphocyte ratios for colorectal liver-only metastases survival. Onco Targets Ther 2017; 10:3789-99.

19. Hirahara T, Arigami T, Yanagita S, Matsushita D, Uchikado $Y$, Kita $Y$, et al. Combined neutrophil-lymphocyte ratio and platelet-lymphocyte ratio predicts chemotherapy response and prognosis in patients with advanced gastric cancer. BMC Cancer 2019; 19(1):672.

20. Ethier JL, Desautels D, Templeton A, Shah PS, Amir E. Prognostic role of neutrophil-to-lymphocyte ratio in breast cancer: A systematic review and meta-analysis. Breast Cancer Res 2017; 19:2. 
21. Fagnoni FF, Zerbini A, Pelosi G, Missale G. Combination of radiofrequency ablation and immunotherapy. Front Biosci 2008; 13:369-81.

22. Janikashvili N, Jayant K, Kikodze N, Mazmishvili K, Pantsulaia I, Sandhu B, et al. Immunomodulatory changes following isolated rf ablation in colorectal liver metastases: A case report. Medicines (Basel) 2019; 6(2):56.
23. Özçelik S, Çelik M, Özçelik M. Evaluation of preoperative neutrophil-lymphocyte ratio in differentiated thyroid carcinoma with lymph node metastasis. J Surg Med 2019; 3:377-80.

24. Feng B, Liang P, Cheng Z, Yu X, Yu J, Han Z, et al. Ultrasound-guided percutaneous microwave ablation of benign thyroid nodules: Experimental and clinical studies. Eur J Endocrinol 2012; 166(6):1031-7. 